\title{
Les conventions entre la France et les peuples marrons du Surinam Contribution à l'étude des middle-grounds post-esclavagistes ${ }^{1}$
}

\section{Yerri Urban}

Maître de conférences en droit public, Faculté de droit de la Martinique, Université des Antilles, Laboratoire Caribéen de Sciences Sociales (LC2S)

\section{Postprint}

L'historiographie récente de l'Amérique du Nord a mis l'accent sur des rapports de forces atypiques entre colons européens et Amérindiens²: dans l'actuel Sud Ouest des Etats-Unis, aux 18e-19e siècles, dans le cadre d'un véritable empire, les Comanches assujettissent des colons et d'autres Amérindiens ; dans la région des Grands Lacs, le Pays d'en Haut, les Algonquiens forment avec les Français, aux 17e-18e siècles, un monde commun que Richard White a baptisé Middle Ground. Ce Middle Ground, avec majuscule, ce monde hybride franco-algonquien, résulte de toutes une série de middle grounds (avec minuscule), d'accords d'un type particulier susceptibles d'être rencontrés ailleurs, à d'autre moments : " un terrain d'entente (middle ground) a pu être trouvé à la suite du choc de valeurs et de cultures résultant de la rencontre entre les Français et les Autochtones. Le résultat est perturbant mais chacun peut penser que les principes fondamentaux de sa société ont été respectés. ${ }^{3}$ ”

On ne se risquera pas ici à affirmer l'existence passée ou présente, en Guyane française, de mondes hybrides, de mondes communs du même type que celui du Pays d'en Haut. II est par contre certain qu'elle a connu des middle grounds, des accords hybrides et syncrétiques manifestant un compromis entre deux systèmes de valeurs, entre deux cultures, traduisant un rapport de force équilibré ou même défavorable au pouvoir colonial. Mais ces accords n'ont pas été conclus, sous une forme écrite, avec des Amérindiens, pour lesquels la règle était l'accord oral. Ils ont été conclus avec les membres d'une population qui s'est constituée dans l'hybridité et le syncrétisme, qui incarne à elle seule toutes les contradictions du Nouveau Monde, amalgamant les figures de l'esclave, de l'autochtone et du colon : les Marrons ${ }^{4}$.

\footnotetext{
1 Cette recherche a été possible grâce au soutien financier apporté par le LABEX CEBA (projet ABIOS). « This work has benefited from an « Investissements d'Avenir " grant managed by Agence Nationale de la Recherche (CEBA, ref. ANR-10-LABX-25-01). »

2 Pekka HÄMÄLÄINEN, L'empire comanche, Toulouse, Anacharsis, 2012 (traduit de l'anglais par F. Cotton) ; Richard WHITE, Le Middle Ground. Indiens, empires et républiques dans la région des Grands Lacs, 1650-1815, Toulouse, Anacharsis, 2009 (traduit de l'anglais par F. Cotton); Thibaut FLEURY, La question du territoire aux Etats-Unis de 1789 à 1914 : apports pour la construction du droit international, thèse de droit, Paris II, 2011 ; Michel MORIN, « Des nations libres sans territoire? Les Autochtones et la colonisation de l'Amérique française du XVle au XVIIle siècle », Journal of the History of International Law 12 (2010), p. 170.
}

3 Ibid. p. 35-36.

4 Sociétés marronnes des Amériques. Mémoires, patrimoines, identités et histoire du XVIle au XXe siècles, Jean MOOMOU \& APFOM (dir.), Matoury, Ibis Rouge, 2015 ; Maroon societies : rebel slave communities in the Americas, Richard PRICE (dir.), New York, Anchor Books, 1973 ; Eric HOBSBAWM, « Postmodernism in the Forest » in ID., On History, New-York, New Press, 1997, pp. 192-200.

Pour une bibliographie plus approfondie, cf. Yerri URBAN, " Le rattachement d'un peuple marron à la France. Les Boni, de la Guyane néerlandaise à la Guyane française (1836-1892) ", Cahiers aixois d'histoire des droits de l'outre-mer français, à paraître. 
La conclusion, par la France, de conventions écrites avec des peuples marrons du Surinam, entre 1860 et 1892, a pourtant tout d'un paradoxe : d'abord parce qu'il s'agit de Marrons du Surinam, qui n'ont ni fui ni attaqué les plantations françaises, ensuite, parce qu'elles sont toutes conclues après la seconde abolition de l'esclavage en Guyane en 1848 , et presque toutes après l'abolition de l'esclavage au Surinam en 1863, enfin, parce qu'en cette seconde moitié du XIXe siècle, on ne s'attend pas à trouver ce type de pratique dans un territoire rattaché depuis longtemps à la France- et officiellement conquis.

Mais ce paradoxe se déploie dans un contexte plus large non moins surprenant. Alors qu'en règle générale, les communautés marronnes qui se sont formées en marge des sociétés de l'Amérique esclavagiste disparaissent ou tendent à s'assimiler grandement après les abolitions de l'esclavage, celles du Surinam non seulement continuent d'exister après l'abolition de 1863, mais se développent : elles donnent naissance à une véritable diaspora, aujourd'hui surtout présente en Guyane française ${ }^{5}$, mais aussi aux Pays-Bas, et dans une moindre mesure en divers endroits de la planète, aussi bien aux Etats-Unis qu'en France métropolitaine. Et cette population continue de croitre : alors qu'ils étaient environ 60000 dans les années 1960, les Marrons du Surinam seraient aujourd'hui 210 000, dont 127000 au Surinam et 67000 en Guyane française ${ }^{6}$.

Six peuples marrons se sont formés au Surinam durant la période esclavagiste, mais deux d'entre eux, les Saramaka et les Ndjuka, seront toujours, et de loin, les plus importants numériquement. Au centre du Surinam, se trouvent les Saramaka, les Matawai et les Kwinti, et à l'Est du pays, sur le Maroni qui marquera rapidement la frontière franconéerlandaise puis franco-surinamaise, se trouvent les Ndjuka, les Paramaka et enfin, les Aluku ou Boni, peuple marron qui a aujourd'hui plusieurs particularités : il a deux noms, le terme Boni étant préféré par l'administration et le terme Aluku par les intéressés7, il est rattaché à la France, alors que les autres peuples demeurent rattachés au Surinam, en tant que colonie néerlandaise puis en tant qu'Etat indépendant.

La dernière particularité des Boni est qu'ils ont conclu la paix avec les Néerlandais en 1860 pour des raisons qui n'ont rien de militaire, bien après avoir cessé d'attaquer leurs plantations, bien après avoir été vaincus par les Ndjuka, en 1793, et peu avant l'abolition de l'esclavage au Surinam de 1863. Trois autres groupes, les Ndjuka en 1760, les Saramaka en 1762, les Matawai en 1762 et 1769, avaient auparavant conclu la paix avec des Néerlandais qui désespéraient de ne pouvoir les vaincre dans des guerres trop couteuses mais comptaient en même temps, par une stratégie de paix séparée, diviser les groupes marrons pour mieux régner. Le traité de paix conclu par les Ndjuka en 1760 a un caractère fondateur : inspiré des traités conclus par la Grande-Bretagne avec les Marrons de la Jamaïque en 1739, il revêt une double dimension, de droit privé et de droit public. La dimension de droit privé, c'est l'affranchissement collectif : les Ndjuka cessent d'être des esclaves évadés et se voient reconnaître la liberté. La dimension de droit public, c'est la reconnaissance des Ndjuka en tant qu'entité politique. Les Ndjuka prennent soin d'assurer leur autonomie durant les négociations, faisant notamment en sorte d'échapper à la justice criminelle néerlandaise. Le traité n'aborde que les questions de compétence en matière criminelle, mais la totale autonomie des Ndjuka dans les autres domaines (c'est-à-dire les autres affaires non-criminelles, les droits d'usage, le mariage, l'héritage et les autres

5R. PRICE, Sally PRICE, Les Marrons, 2ème éd., La Roque d'Anthéron, Vents d'Ailleurs, 2004 (1ère éd. 2003).

6 R. PRICE, « The Maroon Population Explosion : Suriname and Guyane », New West Indian Guide, 87 (2013), pp. 323-327.

7 Cet article traitant des accords conclus avec la France, j'utiliserai le terme «Boni». 
aspects de la vie sociale) sera, de facto, reconnue ${ }^{8}$. II en ira de même pour les Saramaka et les Matawai. Lors des renouvellement des traités dans les années 1830, l'intervention des juridictions néerlandaises en matière criminelle sera plus marquée. En concluant la paix avec les Néerlandais, ces groupes marrons deviennent également leurs alliés. On les appelle des «Marrons pacifiés ». Cette alliance a une dimension militaire, mais les Marrons pacifiés s'engagent également à accomplir une tache plus quotidienne : restituer aux Blancs les esclaves évadés qui seraient réfugiés chez eux, moyennant une prime. Ces Marrons pacifiés s'engagent également, en plus de cesser d'attaquer les plantations, à se tenir à une distance respectable de la zone des plantations.

Pour les Néerlandais, le prix de la paix est le versement régulier d'un tribut, composé essentiellement de biens manufacturés que les Marrons se procuraient auparavant par des raids dans les plantations, pour compléter les échanges commerciaux très réglementés qu'ils ont avec la côte. II est impossible d'ignorer la dimension symbolique de ce tribut : c'est une humiliation pour les Néerlandais, comme le constatent les observateurs de l'époque. II préfèreront d'ailleurs éviter le terme de tribut et de parler de cadeaux ou de présents. Dès qu'ils autoriseront les Marrons pacifiés à accéder librement à la côte (1856), ils remplaceront le tribut par le versement d'une allocation aux chefs de chaque peuple marron pacifié, les Gran Man, en 1857. L'abolition de l'esclavage au Surinam se profile déjà, et les autorités coloniales comptent sur les Marrons pour constituer une nouvelle source de main d'oeuvre.

Du coté français, on souhaite également mettre en valeur la Guyane. Faire venir des Marrons est moins couteux que de faire venir des Bagnards et des engagés ${ }^{9}$. C'est donc dans un but migratoire que la France va conclure des conventions écrites et orales avec trois peuples marrons, les Ndjuka, les Boni et les Saramaka, trois des quatre peuples marrons aujourd'hui présents sur le territoire de la Guyane. Le quatrième peuple, les Paramaka, n'a jamais conclu de convention avec la France ; il est également, avec les Kwinti, un des deux peuples marrons qui n'ont jamais conclu de traité avec les Néerlandais. Est-ce vraiment un hasard?

Ces accords écrits et oraux ont en commun d'être des middle grounds : ils traduisent un rapport de force équilibré voire favorable aux Marrons du Surinam et réalisent un compromis hybride entre deux systèmes de valeurs. Pour cette raison, ces accords vont avoir des effets distordus par rapport aux intentions initiales.

Ces conséquences, plus ou moins inattendues, seront propres à chaque peuple : les autorités coloniales ne s'attendaient pas à reconnaître l'indépendance des Ndjuka (I), à rattacher les Boni à la France (II) et, si elles entendaient bien encadrer l'immigration des Saramaka (III), ce ne sera pas de la manière prévue.

\section{Reconnaître l'indépendance des Ndjuka}

Aujourd'hui peu présente dans les mémoires, la convention franco-ndjuka de 1860 est celle qui se rapproche le plus d'un véritable traité. Bien que largement devenue sans objet par la suite, elle n'en demeure pas moins fondamentale pour comprendre les rapports entre la France et les peuples marrons. Alors qu'au XIXe siècle les « Nations Indiennes », déjà regardées comme "sauvages », perdent leur qualité d'Etat au regard du droit international public par l'introduction progressive d'une condition de « civilisation », la situation particulière des peuples marrons au regard de l'esclavage va favoriser une reconnaissance à contre-courant. Si, en 1836, la France avait préféré refuser de négocier

\footnotetext{
8 Sur les traités et la justice dans un contexte colonial, cf. Bernard DURAND, Introduction historique au droit colonial, Paris, Economica, coll. « Corpus Histoire du droit », 2015, p. 244ss.

9 Pour une partie des sources étudiées, cf. Jean HURAULT, Monique POULIQUEN, Documents pour servir à I'histoire des Noirs réfugiés Bonis de la Guyane française, Paris, 1954.
} 
directement avec les Ndjuka parce qu'elle maintenait l'esclavage, en 1860, bien que la condition de civilisation s'affirme, elle traite directement avec eux parce que l'esclavage est aboli depuis 1848. La reconnaissance abolitionniste d'une « tribu indépendante » $(B)$ succède ainsi au refus esclavagiste de sa reconnaissance $(A)$.

\section{A) La convention franco-néerlandaise de 1836 : le refus esclavagiste de la reconnaissance}

La convention de 1836 est la conséquence d'une double découverte des autorités françaises : d'une part, celle de la domination des Ndjuka sur le Maroni, traditionnellement considéré comme la limite entre les Guyanes française et néerlandaise, d'autre part, celle de la présence des Boni en amont, dans une partie encore plus inexplorée du fleuve, le Lawa, qui s'avèrera par la suite en être le cours supérieur.

Le massacre de ces derniers par les Ndjuka en 1793 avait mis fin aux guerres qu'ils menaient contre les Néerlandais depuis 1768. Par la suite, lors du renouvellement des traités avec les Ndjuka en 1809 puis en 1837, les autorités du Surinam reconnaissent aux Boni un statut particulier : bien qu'esclaves rebelles, il peuvent être en contact avec les Ndjuka, qui doivent les empêcher d'accéder à la zone des plantations et d'attaquer les blancs.

Ceci conforte la domination sur le Maroni des Ndjuka, qui commercent avec la côte et sont aussi présents dans la zone des plantations. Etablis sur le Tapanahoni, le principal affluent du Maroni, et surtout à la confluence entre Lawa et Tapanahoni, les Ndjuka font en sorte d'être les seuls intermédiaires commerciaux entre les populations situées en amont, Boni et Amérindiens, et les populations situées en aval. Ils instaurent pour ce faire un véritable blocus à la confluence, que les Boni cherchent à contourner entre 1836 et 1841 .

En 1836, un explorateur français, Leprieur, est accusé d'avoir conclu un traité avec les Boni, entrainant les protestations des Ndjuka et des autorités néerlandaises. II s'en suivra la signature d'une convention entre les gouverneurs des deux Guyanes datée du 9 novembre 1836, négociée et signée, du côté français, par le procureur général, Vidal de Ligendes. L'article 1er désavoue Leprieur.

Les articles suivants précisent : «Art. 2 : Si des nègres Bonni se présentent sur le territoire français, il leur sera donné connaissance de ce désaveu, et ils seront sommés de se retirer sur le territoire où ils sont tolérés sous la domination des nègres d'Auca. Art. 3 : Le gouvernement de la Guyane hollandaise en faisant connaître aux nègres d'Auca le présent désaveu, leur fera connaître également, ainsi qu'à tous les autres qui sont sous sa domination et qui habitent le Marony que le territoire situé sur la rive droite du Marony à partir de la source de ce fleuve appartient à la France, depuis bien avant qu'ils y fussent établis, que ledit fleuve est la limite entre les deux nations françaises et hollandaises et qu'en conséquence les sujets français ont le droit de passer sur ledit fleuve du Marony. Le Gouverneur de la Guyane hollandaise enjoindra en conséquence aux nègres dépendant de sa domination et établis sur le Marony de respecter la vie et les propriétés de tout Français qui passera sur ce fleuve, sauf, si quelque Français pouvait être la cause de quelque trouble à le conduire à Paramaribo afin qu'il en soit référé aux deux gouvernements. ${ }^{10}$ »

Le texte sera approuvé par le ministère des colonies et par le ministère des affaires étrangères en 1837 .

\footnotetext{
10 Convention entre les Gouverneurs des Guyanes hollandaise et française concernant les nègres Bonis. "Documents... », op. cit. n.9, p. 217. Extrait d'une convention entre les Guyanes française et hollandaise, trouvée dans les archives de Mana, ANOM, FM, SG, GUY39, Dossier D3 (01). L'article 4 a une portée plus limitée.
} 
Alors que les nouveaux traités de paix interdisent notamment aux «Marrons pacifiés 》 d'entrer en relation avec un pouvoir étranger, la convention franco-néerlandaise de 1836 renforce de toute évidence la position des autorités du Surinam. Refusant de reconnaître directement les peuples marrons et donc de négocier avec eux, la France ne fait qu'entériner le discours que lui a tenu le gouverneur néerlandais : les «nègres d'Auca», comme les autres «marrons pacifiés", sont sous la domination du "gouvernement de la Guyane hollandaise». Désormais, le blocus ndjuka se voit complété par un blocus français empêchant les Boni d'accéder à la côte guyanaise. De fait, la convention de 1836 paraît emprunter aux nouveaux traités de paix que les Pays-Bas sont en train de conclure avec les trois peuples marrons " pacifiés " l'idée de cantonnement- sauf que les Boni se voient strictement interdire toute sortie du territoire sur lequel ils sont tenus de rester : ils ne doivent pas se présenter sur le « territoire français » mais demeurer "sur le territoire où ils sont tolérés sous la domination des nègres d'Aucas".

La France entérine la présence des Ndjuka, rattachés aux Pays-Bas, sur les deux rives du Maroni. Dans le même temps, la souveraineté française sur la rive droite du Maroni jusqu'à la source du fleuve est reconnue, le principe de la liberté de navigation et de circulation est affirmé pour les Français. Les Ndjuka doivent respecter « la vie et la propriété " des Français comme ils doivent respecter celle des Blancs au Surinam.Toutefois, en cas de trouble causé par un Français, les Ndjuka doivent l'amener à Paramaribo pour en référer aux deux gouvernements. Mais la France n'a alors aucun établissement sur le fleuve.

Les Boni se retrouvent ainsi enfermés dans une situation singulière : du côté du Surinam, on ne veut envisager de traité de paix, et par là même la reconnaissance de l'autonomie et de la liberté ; du côté de la Guyane, on les renvoie à la domination indirecte du Surinam. Les deux puissances coloniales s'accordent pour considérer qu'ils sont placés sous la domination des Ndjuka - ou plus exactement que les questions les concernant relèvent avant tout de la compétence des Ndjuka. Les autorités françaises paraissent ainsi privilégier leurs préjugés esclavagistes par rapport à leurs intérêts stratégiques : la frontière du Maroni est confirmée, mais elles préfèrent reconnaître une forme de domination des Pays-Bas sur le fleuve via leur domination supposée sur les Ndjuka plutôt que de négocier directement avec ce peuple marron.

Si elle ne la confirme pas juridiquement, cette convention confirme pourtant politiquement la puissance des Ndjuka. Les Néerlandais sont leurs porte-parole auprès des Français et ces derniers, par leur blocus, contribuent à la domination ndjuka sur le Haut-Maroni. C'est lorsque les Ndjuka seront affaiblis que la France traitera directement avec eux.

\section{B) La convention franco-ndjuka de 1860 : la reconnaissance abolitionniste d'une « tribu indépendante »}

La convention franco-ndjuka du 11 septembre 1860 demeure un texte assez énigmatique : on ne dispose ni de source sur son élaboration, ni du texte original. Seule une copie nous est parvenue.

Le contexte a changé depuis 1836 : l'esclavage a été aboli en 1848 en Guyane française et le sera en 1863 au Surinam. Les Français sont en train de s'établir sur le Maroni et sont importunés par les Ndjuka, qui les empêchent notamment d'accéder au Haut-Maroni et à une main d'oeuvre boni. Les Néerlandais vont s'efforcer de mettre fin à cette source de tensions diplomatiques en facilitant un accord entre Ndjuka et Français, accord comprenant notamment la libre circulation sur le Maroni et la fin de la "tutelle " ndjuka sur les Boni. Les Néerlandais acceptent ainsi officieusement de laisser les Français négocier directement avec les peuples marrons du Surinam, commercer avec eux, les inciter à émigrer en Guyane, bref, de laisser ces Marrons avoir un existence transfrontalière reconnue par les deux puissances de manière analogue à ce qui se 
pratique déjà avec des peuples amérindiens qui, du coté français, ne peuvent en principe être réduits en esclavage.

De plus, Néerlandais et Français croient alors que le Tapanahoni est le cours supérieur du Maroni : il est logique d'organiser la présence des Ndjuka sur ce qu'on suppose alors être la rive française, c'est à dire la rive droite du Tapanahoni et la confluence entre le Lawa et le Tapanahoni. Les Français espèrent que les Ndjuka préféreront de ce fait commercer avec leurs établissements, mais espèrent aussi convaincre les Boni d'émigrer dans l'Approuague, fleuve situé à l'Est de Cayenne. Ce sera dans les deux cas un échec. En effet, dès novembre 1860, les Néerlandais mais aussi les Français comprendront que c'est en réalité le Lawa qui joue le rôle de cours supérieur du Maroni. De ce fait, la convention jouera un rôle pratique plus modeste que prévu. Elle n'en démontre pas moins qu'on a changé de monde : à un consensus esclavagiste franco-néerlandais détournant les yeux des réalités d'un terrain marginal succèdent des rivalités entre puissances européennes, des litiges frontaliers, la volonté de s'accaparer les faveurs des peuples marrons, des jeux d'alliances et de contre-alliances.

Le 8 septembre 1860 a lieu à Albina, le poste surinamais situé en face de Saint-Laurent du Maroni, une rencontre entre le gouverneur du Surinam, Van Landsberge, le chef d'étatmajor du gouverneur de la Guyane Tardy de Montravel, Sibour, et le Gran Man des Ndjuka, Beeiman. Les représentants des deux colonies obtiennent de Beeiman qu'il accepte de laisser passer les Français et leurs «alliés», qu'il accepte le principe de libre circulation sur le fleuve, notamment pour les Boni. Peu après, à partir du 9 septembre, les Français négocient séparément avec les Ndjuka et concluent avec eux, le 11 septembre 1860 , des «arrangements» signés au pénitencier de Saint Louis, et qui seront par la suite approuvé par Tardy de Montravel11. Le texte est antidaté au 8 septembre... ce qui est plutôt scrupuleux au regard des pratiques coloniales. Le texte est la première convention signée par la France avec un peuple marron :

«Art. 1er. Le Grand Man Byman, éclairé par S.E. le gouverneur de la Guyane hollandaise en séance publique et en présence de ses capitaines, reconnaît l'entière liberté du fleuve Maroni comme de ses affluents ; c'est à dire qu'à partir de ce jour, 8 septembre 1860, toute personne appartenant à quelque nation, tribu, peuplade que ce soit, Française, Auca, Bonie, Roucouïenne, etca, peut remonter ou descendre le Maroni ou ses affluents pour raison de commerce ou toute autre sans que nul n'y puisse porter le moindre empêchement ou obstacle.

Art. 2. Le Grand man Gouverneur des Aucas reconnaissant des assurances de bon voisinage données au nom de S.E. le gouverneur de la Guyane française s'engage dans la mesure de ses forces et de son autorité à accorder aide et protection à tout sujet français ou allié, séjournant ou voyageant sur les terres où il commande et, en réciprocité, aide et protection sont garanties à tout Auca ou allié des Aucas voyageant et séjournant sur les terres soumises à la domination française.

Art. 3. Le gonflement en usage jusqu'à ce jour ne pourra plus être pour les Français qu'une épreuve librement consentie puisque, à partir de ce jour, S.E. le gouverneur de Cayenne entrant en relations amicales avec le Grand man des Aucas se charge de sévir suivant nos lois, contre tout sujet Français ou protégé qui se rendrait coupable d'un méfait quelconque vis-à-vis du Grand man ou de l'un de ses sujets, tout comme le Grand man aurait à sévir contre tout Auca qui se rendrait coupable d'un méfait quelconque vis-à-vis d'un sujet Français ou protégé de la France.

Art. 4. De part et d'autre des relations commerciales seront favorisées autant que possible.

${ }_{11}$ Arrangements convenus et librement consentis de part et d'autre entre le Grand man Byman chef des Nègres Bosch Aucas et Monsieur Sibour, lieutenant de vaisseau, chef d'état-major de S.E. le Gouverneur de la Guyane française et son fondé de pouvoir (copie). Ibid. 
Art. 5 . Pour preuve de son bon vouloir et de ses intentions amicales, chaque fois qu'il aura pu être prévenu, le Gran man enverra auprès des voyageurs officiels se rendant auprès de lui, des guides et des canots.

Art. 6. Le Gran man des Aucas étant l'ami du Gran man Français, il sera accordé une prime de 20 francs pour tout transporté déserteur de nos établissements pénitentiaires capturé et ramené sur un de ces établissements.»

Les autorités coloniales publieront le texte dans la Feuille de la Guyane française, le supplément pour la Guyane du Moniteur universel... mais dans sa partie non officielle : reprenant la publication issue du rapport de Sibour, déjà parue dans la Revue maritime et coloniale, elles laissent figurer le traité en note en bas de page, amputé de son article 6, où la mention d'un "Gran man Français " a pu choquer. Mais les Français ne s'en considèrent pas moins liés par le texte : Tardy de Montravel transforme l'article 6 , seule disposition à portée financière, en décision publiée quant à elle au Bulletin officiel de la colonie $^{12}$. Le Gouverneur français sollicite par ailleurs l'assentiment du ministre pour cette convention qui pourra également produire par la suite une certaine irritation du coté néerlandais.

Si les autorités françaises ne paraissent pas reconnaître de valeur juridique à cet accord, elles se considèrent de toute évidence comme politiquement liées.

Ceci rend ces « arrangements " d'autant plus précieux : ils rendent lisibles les principes qui sous tendent les pratiques qui vont régir, de fait, la situation des peuples marrons du Surinam en Guyane française.

Le paradoxe apparent de ce texte lié à la volonté de la France de voir sa souveraineté devenir effective sur la rive droite d'un Maroni dont le Tapanahoni serait le cours supérieur, est qu'il reconnaît aux Ndjuka le statut d'entité politique indépendante. Les propos de Tardy de Montravel sont clairs : il évoque les « tribus Boschs, Bonis et autres qui habitent le haut du Maroni et les bords de ses affluents supérieurs, tribus qui, pour la plupart, sont liées par des conventions avec le gouvernement hollandais " et qualifie les Ndjuka de " tribu indépendante ${ }^{13}$. Ce discours prend entièrement le contrepied de la reconnaissance d'une domination néerlandaise sur les peuples marrons en 1836 : cette fois, il leur arrive juste d'être liés par des conventions avec les Pays-Bas, comme n'importe quel Etat indépendant, ce qui ne les empêche donc pas de conclure des conventions avec les gouvernements d'autres Etats.

La finalité pratique de cette conception ne fait guère de doute : c'est le seul moyen pour la France de justifier le fait qu'elle traite directement avec les peuples marrons, au lieu de traiter avec les seules autorités néerlandaises, comme elle l'avait fait auparavant. Mais elle formule aussi les pratiques existantes à l'égard des peuples amérindiens, dont les autorités françaises sont plus familières : les " tribus indépendantes " ne sont pas seulement marrons, c'est la seule situation particulière des Ndjuka qui appelle la conclusion d'une convention écrite. S'agissant de ces derniers, cette conception entérine aussi, tout simplement, un rapport de force : les Français ont pu constater la réalité de la puissance ndjuka sur le Maroni et le caractère tout relatif de l'autorité que les Néerlandais prétendaient exercer sur eux. On voit ainsi se dessiner ce que qui sera la ligne des autorités françaises : considérer les peuples marrons comme des «tribus indépendantes", leur reconnaître en conséquence une plus grande autonomie qu'au Surinam, ce qui peut être une manière d'être attractif en terme migratoire, mais aussi de conclure des accords avec les Gran Man concernant la main d'oeuvre marron, avec leurs règles propres.

12Décision qui fixe la prime de capture des transportés évadés à l'égard des nègres awcas, 21 août 1861, BOGF 1861, n 329, p. 328.

13 Lettre du Gouverneur de la Guyane française au Ministre de l'Algérie et des colonies du 3 septembre 1860, ANOM, FM, SG, GUY39, Dossier D3 (01). 
Cette indépendance n'implique pas la reconnaissance d'une souveraineté territoriale : là, le territoire est français, et clairement français, ce qui n'est pas sans évoquer l'attitude à l'égard des Nations Indiennes d'Amérique du Nord. La pratique française est assez complexe : elle ne conteste pas la présence des peuples marrons sur les terres qu'ils occupent traditionnellement, généralement situées sur le domaine privé de l'Etat, mais elle aura tendance à leur accorder des concessions dès lors qu'il s'agit d'occuper de nouveaux territoires. L'indépendance est donc avant tout entendue du strict point de vue personnel : le peuple marron est reconnu en tant que tel, mais il n'a pas officiellement son propre territoire.

Le texte est d'ailleurs délibérément flou à ce sujet : il reconnait l'autorité du Gran Man ndjuka sur les terres où son peuple est présent («terres où il commande», art. 2), sans préciser dans quelle colonie elles sont situées, tout en renvoyant à une zone où l'autorité française est effective («terres soumises à la domination française», art. 2). II ne s'agit surtout pas d'en dire plus, même si, pour les Français, le Tapanahoni devant constituer le cours supérieur, les Ndjuka sont présents sur les rives françaises et néerlandaises aussi bien sur le Bas que sur le Haut Maroni.

L'article 3 pose le principe d'une stricte compétence ratione personae en matière pénale, qui va au-delà de ce que reconnaissent les traités néerlandais des années 1760 (sans parler de ceux des années 1830) : tout crime ou délit («méfait quelconque») commis par un Français ou protégé français (ressortissant français) à l'égard d'un Ndjuka relève de la compétence de la France, tandis que tout crime ou délit commis par un Ndjuka à l'égard d'un Français ou protégé français relève de la compétence ndjuka. Ce privilège de juridiction est sans doute le produit de la situation de fait existant en Guyane aussi bien pour les peuples amérindiens que pour les peuples marrons. Elle se situe également dans le prolongement des pratiques en Amérique du Nord aux XVIle-XVIIle siècle, où la justice française ne parvint pas à s'imposer aux Amérindiens, alors que la volonté de « civiliser juridiquement» les Amérindiens s'imposera à partir de la fin du XIXe siècle aux Etats-Unis et au Canada. Nous sommes à l'exact opposé des principes qui régissent les rapports entre la France et ses indigènes dans les territoires où cette catégorie existe, mais aussi des rapports de la France avec les nationaux des pays de Capitulations : à l'opposé de l'inégalité justifiée par la civilisation, par la supériorité du droit occidental, par la mission civilisatrice par le droit. Ici, chacun juge ses ressortissants comme il l'entend, alors que là-bas c'est la juridiction française qui est toujours compétente.

Les Français pourront bien tenir, à l'occasion, un discours civilisateur vis-à-vis des Marrons, et continueront de les regarder comme des « sauvages » ou des «primitifs». Le critère de la civilisation n'est pas ici déterminant : du point de vue juridictionnel, ils sont traités sur un pied d'égalité, à la différence, par exemple, de l'Empire ottoman ou du Japon à la même époque. Chacun est compétent pour sanctionner les crimes et délits commis par ses propres ressortissants : le principe est simple, et paraît avoir été longtemps appliqué ${ }^{14}$. Ce principe implique aussi la totale autonomie des Marrons d'un point de vue «judiciaire» et d'un point de vue «législatif» : comme au Surinam, le silence implique l'autonomie. C'est ce qui empêche notamment de considérer les Marrons comme étant dotés d'un statut identique à celui des indigènes de l'Empire colonial : leur relation avec la France ne repose pas sur une inégalité de principe.

L'entité politique ndjuka, reconnue jusqu'alors par les seuls Néerlandais est désormais reconnue de facto par les autorités françaises, et ce, de manière malgré tout beaucoup plus officielle qu'un peuple amérindien au même moment : ils vont se voir reconnaître une

\footnotetext{
${ }_{14} \mathrm{Si} \mathrm{l'on} \mathrm{trouve} \mathrm{très} \mathrm{rarement} \mathrm{mention} \mathrm{de} \mathrm{plainte} \mathrm{de} \mathrm{Marrons} \mathrm{contre} \mathrm{des} \mathrm{orpailleurs} \mathrm{Français} \mathrm{ou} \mathrm{étrangers}$ provoquant l'intervention des autorités françaises, on ne trouve pas en sens inverse de plainte contre des Marrons provoquant le même type de réaction. Pour une adaptation de ces principes aux Saramaka, cf. infra.
} 
existence intercoloniale, interimpériale. II est inconcevable désormais que les Français remettent en cause l'autorité du Gran Man ndjuka sur ses sujets et sur ses capitaines même s'ils sont établis sur le sol français. Aujourd'hui encore, il est inconcevable que la France désigne ou fasse désigner un Gran Man ndjuka pour les seuls Ndjuka établis en Guyane française.

Enfin, ce ne sont plus les captures d'esclaves marrons -comme dans les traités conclus avec les Néerlandais- qui sont rémunérées, mais celles de bagnards évadés (art. 6). Cette disposition sera longtemps appliquée ${ }^{15}$.

Dernière entorse aux principes en vigueur à l'époque : au regard du droit international public, ceux qu'on nomme alors les « peuples sauvages » ne se voient reconnaître aucune existence internationale à partir de la deuxième moitié du XIXème siècle, approche qui trouvera sa consécration avec l'acte final de la conférence de Berlin signé le 26 février 1885. C'est à cette dernière catégorie que les autorités coloniales ont spontanément tendance à assimiler les peuples marrons. Or la France ne va pas les traiter comme des «peuples mineurs», donc incapables, mais comme des « nations libres sans territoire ", comme des «tribus indépendantes », des peuples indépendants, certes sans territoire propre, mais en les plaçant sur un pied d'égalité avec les autorités de la colonie («Gouverneur des Aucas», «gouverneur de la Guyane française» ; «Gran Man des Aucas», "Gran Man Français»). Dans ce contexte, la nation française fait partie des nombreuses «nations, tribus, peuplades» de la région du Maroni, à côté de celles des «Aucas, Bonis, Roucouïennes, etca» (art. 1er).

Un compromis hybride a ainsi été trouvé. Il y a sans doute également ici de la part des autorités françaises la volonté d'avoir des pratiques relativement homogènes quant aux Marrons et aux Amérindiens. II n'en demeure pas moins que l'exclusion des Marrons, comme des Amérindiens, de l'application du droit de la nationalité française, la reconnaissance de pseudo-nationalités marronnes et amérindiennes perdureront jusqu'à très récemment, alors que le principe selon lequel il faut éviter l'apatridie des colonisés est progressivement admis dans le reste de l'Empire colonial16.

Fin 1861, une nouvelle expédition franco-néerlandaise confirme que c'est le Lawa, et non le Tapanahoni, qui constitue le cours supérieur du Maroni. La France préfèrera alors une frontière incertaine à un règlement de litige qui paraît très mal engagé. Les Boni, tout comme les Ndjuka, se trouveront donc établis en partie dans la zone située entre le Tapanahoni et le Lawa, le contesté franco-néerlandais, considéré comme neutre tant que le litige n'est pas tranché. Cette situation, qui fait des deux peuples marrons les premiers occupants de ce territoire, maintient un rapport de force favorable, surtout pour les Boni qui obtiendront leur rattachement à la France.

\section{Rattacher les Boni à la France}

Si les grands principes qui régissent les relations des autorités françaises avec les peuples marrons sont posés par la convention de 1860, de nombreux éléments seront précisés plus tard, notamment entre 1870 et 1900. La présence des Boni sur la limite française du contesté, puis la réactivation du conflit frontalier à partir de 1885 vont favoriser la revendication d'une alliance franco-boni destinée à faire contrepoids à l'alliance néerlando-ndjuka et, partant, la naissance de principes complémentaires régissant les rapports des autorités françaises avec les autorités marrons.

15 Gérard THABOUILLOT, Un projet politique et administratif pour l'arrière-pays de la Guyane française : le territoire de I'Inini (1930-1969), Thèse d'histoire, Paris IV, 2012, p. 203.

16 Y. URBAN, “Empire colonial et droit à la nationalité »Droits de l'homme et colonies, Alexandre DEROCHE \& Eric GASPARINI (dir.), Aix-en-Provence, PUAM, à paraître ; Catherine BENOÎT, « «Pampila » et politique sur le Maroni : de l'état civil sur un fleuve frontière ", Histoire de la justice, nº 26. 
L'influence des pratiques en vigueur au Surinam est nette, ce qui n'exclut pas celle des pratiques françaises à l'égard des Amérindiens.

Certaines pratiques importées du Surinam vont être en partie imposées à la France par les Boni : un uniforme est réclamé par le Gran Man en 1862, et à nouveau dans les années 1880 ; l'allocation, mise en place en 1857 au Surinam pour remplacer le tribut, commencera à être versée à partir de 1877 au Gran Man Anato. L'investiture des autorités marrons par la France, où l'on voit le modèle surinamais mais aussi le modèle amérindien, commence avec l'investiture du capitaine boni Apatou par un arrêté du gouverneur du 26 juillet 1887. Enfin, le principe selon lequel c'est le lieu de résidence du Gran Man qui détermine l'Etat auquel il se rattache prend naissance suite au règlement du conflit frontalier en faveur des Néerlandais par l'arbitrage du tsar Alexandre III, le 25 mai 1891. En 1892, le Gran Man des Boni Ochi préfère s'établir sur la rive droite du Lawa plutôt que de se mettre « sous la protection » des Pays-Bas.

Dans ce processus de construction de l'alliance franco-boni, qui donnera à ces derniers un rôle d'interlocuteur marron privilégié, deux accords vont jouer un rôle important : un accord oral conclu dans les années 1870, qui se traduira par la reconnaissance symbolique de cette alliance $(A)$ alors que sa reconnaissance officielle se traduira par un accord écrit et publié conclu en 1887 (B).

\section{A) L'accord oral des années 1870 : la reconnaissance symbolique du lien franco- boni}

Par le biais d'un processus original, où le jeu entre l'oral et l'écrit produit de multiples infléchissements, cet accord va aboutir à l'importation de pratiques qui marquent au Surinam la reconnaissance des autorités marrons : le versement d'une allocation et l'attribution d'un uniforme.

Le contenu de cet accord est décrit pour la première fois en 1878, dans une correspondance administrative17: les autorités coloniales avaient promis au prédécesseur du Gran Man Anato, Atiaba, " une gratification mensuelle de cent francs payable sur les fonds du service local s'il voulait s'engager à prêter assistance aux explorateurs du Haut Maroni, notamment en leur fournissant des guides et des pagailleurs pour un salaire journalier de $3 \mathrm{f}$. au maximum, salaire restant bien sûr à la charge des employeurs ". Le voyage d'exploration de Jules Crevaux en 1877 donne l'occasion à Anato de " ratifier les promesses de son prédécesseur en aidant » cette expédition. II reçoit en conséquence le premier terme de la « gratification ». Le commandant supérieur du Maroni, chargé de s'occuper du versement, doit s'assurer que le Gran Man Anato respecte ses engagements. Dès cette époque, cet accord oral " restitué » par écrit peut -être présenté comme une « convention », ce qui laisse croire à l'existence d'un accord écrit.

Une «note sur les Bonis, sous toutes réserves»18, non datée et non signée, rédigée sans doute entre 1882 et 1886 par l'administration coloniale de Cayenne, donne par la suite une tournure plus solennelle à la « sorte de convention » qui aurait été conclue en 1878 par la France et le « Grand-Man des Bonis »: le Gran Man boni tend à y devenir un équivalent français des Gran Man reconnus par les Néerlandais, qui se voit attribuer, en plus de l'allocation, un uniforme ; ses obligations deviennent plus vagues. L'auteur indique que «la nation Boni a toujours été l'alliée de la France» et précise qu' «En 1878, une sorte de convention fut conclue entre la France, représentée je crois par le commandant supérieur de Saint-Laurent, d'une part, et le Grand-Man des Bonis de l'autre. Il était stipulé

\footnotetext{
17 Lettre du 9 février 1878 du directeur de l'intérieur au commandant supérieur du Maroni, ADGuyane, X352. Cf. aussi Note du directeur de l'intérieur au directeur de l'administration pénitentiaire du 8 février 1878, AD Guyane, X388.
} 
de première part savoir $1^{\circ}$ Que le Grand-Man recevrait de la France une pension annuelle de 1200f; $2^{\circ} Q u$ 'il lui serait donné un costume chamarré d'or à l'imitation de celui que les Hollandais donnent au Grand Man des Bosh; $3^{\circ}$ Que lorsqu'il descendrait à Saint Laurent en grand appareil (c'est à dire entouré de nombreuses pirogues et au son du tambourin), il serait reçu officiellement avec salves de coups de fusils etc... Et de seconde part savoir : Que moyennant l'exécution de ces promesses, le Gran Man favoriserait la descente et l'établissement des Bonis vers le bas du fleuve, ferait bon accueil aux voyageurs sur son territoire enfin que les prix des journées de canotages, tâches etc... seront fixés invariablement sauf recours au Gran Man lors d'inexécution de la part de ses sujets. Des documents écrits attestant cette convention existent à Saint Laurent. ${ }^{19}$ "

Le principe du versement de l' allocation par la France au Gran Man paraît dès lors admis. II semble que l'uniforme a, quant à lui, posé plus de difficultés. L'importance de l'oralité et les différentes difficultés de communication aboutissent à la transformation de cet accord oral en une série de conventions écrites imaginaires, ce qui débouchera sur l'adoption d'un accord écrit bien réel cette fois.

\section{B) L'accord franco-boni de 1887 : la reconnaissance officielle de l'alliance franco-boni}

C'est à la faveur du réveil du conflit frontalier que les obligations du Gran Man Anato à l'égard de la France vont prendre un tour encore plus vague, rendant par là même leur lien plus abstrait. En effet, la découverte, en 1885, sur le contesté, en plein pays boni, d'un gisement d'or, rend nécessaire le règlement du litige. En 1887, suite à une lettre, peut-être apocryphe ${ }^{20}$, d'Anato, datée du 2 janvier, faisant part de conflits avec des chercheurs d'or, les autorités françaises souhaitent interdire l'exploitation de l'or sur le contesté, neutre jusqu'à ce que sa situation soit tranchée.

Le 21 janvier 1887, le gouverneur Le Cardinal demande au directeur de l'intérieur de solliciter les autorités de Saint-Laurent du Maroni pour trouver «la convention passée en 1861 avec le chef des Bonis auquel des terrains avaient été concédés dans le HautMaroni en représentation de certaines clauses que le Grand Man devait exécuter loyalement. La protection de nos nationaux était la principale condition de cet acte qui maintenait bien entendu la souveraineté de la France sur la rive gauche de l'Awa et celle du Maroni. » II l'a fait chercher en vain à Cayenne. ${ }^{21}$

La réponse du commandant supérieur du Maroni, le 14 février 1887, est claire : la seule convention datant des années 1860 dont il a trouvé trace est celle conclue le 8 septembre 1860 avec les Ndjuka. Le seul document définissant les rapports avec les Boni est «une décision sans date (...) qui a trait aux engagements du Gran Man vis-à-vis de l'administration locale et vice versa" (peut-être la note non datée citée supra). De l'ensemble des décisions qu'il a pu se procurer, «il ressort que les bonis et les boschs sont indépendants, puisqu'on a traité avec eux et qu'il a été alloué une indemnité au gran man pour le concours que ses sujets prêteraient à nos nationaux».22

Les autorités coloniales de Guyane prennent alors la décision de se prévaloir auprès de leur ministre de tutelle d'un traité daté de 1861, dont les termes supposés rappellent ceux de la convention franco-ndjuka de 1860 (art. 2) : le Gran Man boni devrait «aide,

\footnotetext{
19 Ibid.
}

20 Tristan BELLARDIE, Les Relations entre Français et Bonis en Guyane française (1836-1893), mémoire, Université de Toulouse-Le Mirail, 1994, p. 87-88.

21 Demande du Gouverneur au Directeur de l'intérieur, 21 janvier 1887, AD Guyane, X388.

22 Lettre du commandant supérieur du Maroni au directeur de l'administration pénitentiaire du 14 février 1887 et pièces jointes, Ibid. 
assistance et protection aux français, en échange de la consécration de son autonomie et de son entière indépendance vis-à-vis du chef des Bosch».23 Mais elles savent désormais qu'elle doivent donner au lien franco-boni une confirmation écrite, tout en interdisant l'exploitation de l'or sur le contesté.

Elles vont pour ce faire conclure une convention par laquelle le Gran Man s'oblige à empêcher l'accès à la rive gauche du Lawa, tout en continuant à se référer à I'hypothétique traité de 1861. En effet, en mars 1887, le gouverneur Le Cardinal rencontre le Gran Man Anato au village qu'a fondé il y a peu de temps l'ancien guide boni de Creveaux, Apatou, sur la rive française. Ils signent un «acte» le 14 mars 1887, lequel sera publié24 : "Le Grand-man Anato s'engage à interdire le passage sur la rive gauche de l'Awa à tous les exploiteurs et chercheurs d'or qui viendraient s'établir sur le territoire contesté entre la France et la Hollande. Conformément au traité de 1861, le Grand-man continuera à prêter aide, secours et assistance sur la rive droite de l'Awa, concernant les terrains régulièrement concédés par la Direction de l'Intérieur à Cayenne».

L'autorité territoriale du Gran Man sur la rive gauche est ici reconnue, mais dans l'attente du règlement du conflit. Le recours à la terminologie de la convention francondjuka de 1860 (par l'intermédiaire du « traité de 1861 ») montre que, dans l'esprit des Français, ils occupent une place analogue à celle qu'aurait occupé les Ndjuka si le Tapanahoni avait constitué le cours supérieur du Maroni. L'alliance franco-boni n'en est pas moins confirmée.

Peu après, la France, qui avait reconnu de nombreuses fois des capitaines amérindiens, investit pour la première fois une autorité boni. Le 26 juillet 1887, un arrêté du gouverneur25 dispose:

"Le Boni Apatou est nommé capitaine de la tribu des Bonis dans le haut Maroni, pour toute la circonscription du saut Hermina, sous la suzeraineté du Grand-Man Anato, et avec le consentement de ce Chef.

Dans cette position, il devra ses bons offices a tous les Français.

Les autorités civiles et militaires fourniront, au besoin, à Apatou, aide, assistance et protection.»

Cet acte unilatéral se singularise par son vocabulaire conventionnel, qui montre la persistance de rapports marqués par l'oralité : si Apatou est nommé capitaine par le Gouverneur, c'est avec l'accord du Gran Man et sous la « suzeraineté » de celui-ci. La suite du texte reconnait une véritable enclave boni en territoire français, comme en contrepartie de la convention signée en mars ${ }^{26}$. La description des obligations réciproques (al. 2 et 3) trahit toujours l'influence de la convention franco-ndjuka de 1860 (art. 2), mais en mettant cette fois l'accent sur les obligations de Français.

23Lettre du Gouverneur de Guyane au ministre de la Marine et des Colonies, 30 mars 1887, « Documents... », op. cit. n.9, p. 201.

${ }_{24}$ Acte intervenu entre le Gouverneur de la Guyane française et le Grand-man des Bonis pour interdire le passage sur la rive gauche de l'Awa à tous les exploiteurs et chercheurs d'or qui viendraient s'établir sur le territoire contesté entre la France et la Hollande, BOGF, 1887, n² 279, p. 185.

25 Arrêté nommant Apatou capitaine des Bonis dans le Haut-Maroni, 26 juillet 1887, BOGF, 1887, $n^{\circ} 676, p$. 652.

26 La France avait concédé une façade de $200 \mathrm{~m}$ de rive sur une profondeur de $4 \mathrm{~km}$ de manière définitive à Apatou et à ses héritiers le 7 septembre 1885, assorti d'une prime de 15F par transporté évadé et arrêté. «Les Relations... », op. cit. n.20, p. 79. 
Le Gran Man ne respectant pas la convention du 14 mars (mais avait-il les moyens de la respecter?), le gouverneur suspendra le versement de l'allocation pour l'exercice $1887^{27}$ : elle n'a plus grand chose à voir avec la rétribution de l'aide aux explorateurs qui la justifiait à l'origine. Elle est désormais un attribut du Gran Man des Boni, alliés des Français, et sa suspension n'est plus que le signe du mécontentement des autorités françaises à son égard.

Ainsi se formalisent les relations franco-boni : par des consensus sur des pratiques et des malentendus quant à leur signification, par des transcriptions erronées, par des exagérations et des oublis. La place de l'oralité tendra par contre à évoluer lorsqu'il s'agit d'encadrer l'immigration temporaire d'un peuple marron dont le territoire traditionnel se situe au centre du Surinam.

\section{Encadrer l'immigration des Saramaka}

Présents en Guyane dès les années 1860, notamment à Mana, les Saramaka vont progressivement assurer la navigation sur les principaux fleuves de la Guyane, la Mana, l'Approuague, l'Oyapock, à l'exception du Maroni28. Cette immigration saramaka trouve elle aussi son origine dans les mesures précédant l'abolition de l'esclavage au Surinam : la fin de la remise du tribut et son remplacement par le versement d'une allocation au Gran Man en 1857 incitent les Saramaka à émigrer vers la côte et à acheter des produits manufacturés avec le fruit de leur travail. Ces gains résultent de leur travail forestier et de la vente de leurs produits agricoles sur la côte. S'y ajoutent après l'abolition néerlandaise de 1863 l'abattage et le transport de bois entre les fleuves Suriname et Saramacca qui fournissent un travail saisonnier au Surinam, justifiant une absence du village de quelques mois consécutifs, et le transport fluvial en Guyane, qui justifie des absences de plusieurs années. Cette émigration temporaire et masculine dans la colonie française va modifier considérablement la société saramaka : d'après Richard et Sally Price, "la plupart des hommes saramaka passaient près de la moitié de leur vie en Guyane " au XXe siècle ${ }^{29}$, revenant approvisionner leurs femmes au village pour qu'elles puissent avoir assez de réserve pendant 4 ou 5 ans. Du coté français, les hommes saramaka, sous l'autorité de leurs capitaines, nommés à partir de la toute fin des années 1890, s'établissent notamment à Mana, Régina, Saint-Georges de l'Oyapock ; ils prennent des femmes créoles. Leurs enfants seront créoles. Comme pour le village boni d'Apatou, les autorités françaises pourront reconnaître de véritables enclaves saramaka avec des concessions et l'autorité d'un capitaine.

Alors que, pour les Français, la conclusion d'accords avec les Ndjuka et avec les Boni devait provoquer un nouveau comportement chez ces derniers, la volonté de conclure un accord avec les Saramaka traduit la volonté d'organiser un comportement qui existait déjà. Mais y a t'il eu une ou deux conventions? Le mythe de la convention écrite est prégnant dans les relations franco-marronnes. De ce fait, l'immigration temporaire des Saramaka sera encadrée par un accord écrit semi-officiel en $1892(A)$ et par la référence à une fausse convention franco-saramaka de 1883 dans les années 1960 (B).

\footnotetext{
27 Cf. lettre du Gran Man Anato au gouverneur de Cayenne du 15 octobre 1887 (AD Guyane, X388) ; Extrait du procès-verbal de la séance extraordinaire du conseil privé, « Documents... », op. cit. n.9, p. 203 ; « Les Relations », op. cit. n.20, p. 90-92.
}

28 « Les Marrons », op. cit. n.5, p. 51- 65.

29 Ibid., p. 56. 


\section{A) Par un accord écrit semi-officiel en $1892^{30}$}

C'est lors d'un déplacement officiel à Cayenne, le 1er avril 1892, que le Gran Man Akoosu conclut un accord avec Adolphe Bally, propriétaire de placer, membre de la commission d'immigration et ancien président du conseil général. Le règlement récent du contentieux frontalier franco-néerlandais, la disparition conséquente du contesté, le choix du Gran Man des Boni de s'établir sur la rive française au même moment expliquent cette solution : les Néerlandais ayant laissé les Boni choisir le rattachement à la France, les autorités françaises ne peuvent pas remettre en cause le rattachement des Saramaka aux Pays-Bas en traitant directement avec leur Gran Man. Elles ne peuvent non plus contourner l'interdiction faite aux «Marrons pacifiés » de traiter avec des puissances étrangères. Elles préfèrent utiliser une personne physique comme prête-nom, mais feront comme si l'accord avait été conclu avec un représentant officiel de la France : faute de pouvoir faire conclure l'accord par un représentant officiel, on le fait conclure par un représentant officieux. Les autorités françaises se considéreront politiquement liées par cet accord.

En voici le contenu :

"Au nom de tous mes capitaines, chefs de village et en celui de tous mes sujets,

Moi, Akrosoe Abraham, Granman de la tribu des Saramacas établis dans la haute Guyane hollandaise, voulant reconnaitre les services rendus aux honneurs de ma tribu par M. Adolphe Bally, citoyen français, ancien président du conseil général, ai conclu avec lui un pacte d'amitié qui se résume dans les dispositions suivantes :

Le Granman Abraham s'engage à assurer tous les transports par canotage qui lui seront demandés par M. Bally, soit pour lui-même soit pour ses amis. A cet effet il mettra à sa disposition tout le personnel qui lui sera nécessaire.

II promet aussi d'envoyer à $M$. Bally des familles de cultivateurs saramacas pour l'exploitation des bois de construction, de la gomme de balata, que pour faire des abattis et plantations de denrées vivrières ou d'autre produits agricoles, dans telles régions $d u$ pays que $M$. BALLY désignera.

M. BALLY prend de son côté l'engagement de continuer (de prêter?31)aux hommes de la tribu des Saramacas son aide et son assistance. II donne au Granman Abraham l'assurance que les lois françaises, protectrices de la liberté du travail et de l'égalité des hommes, n'apporteront aucune entrave à la libre exécution du présent traité fait sous la garantie de la bonne foi respective des parties contractantes. "

Bally ajoute dans une note annexée à l'accord : "Pour arriver à conclure ce traité j'avais du faire de nombreux cadeaux au Granman des Saramacas, entre autre chose une jolie petite pièce de canon avec de la poudre. "

S'agissant des obligations saramaka, l'esprit du texte n'est pas très différent de celui de l'accord oral franco-boni des années 1870 , même si les services qui doivent être rendus en Guyane sont ici plus larges. C'est surtout la contrepartie qui diffère : Bally reconnaît l'autorité du Gran Man sur les Saramaka établis sur le territoire de la Guyane française, et l'existence de véritables enclaves saramaka en territoire français. S'il ne peut y avoir ici d'allocation en sus de celle déjà versée par les Pays-Bas, le texte se situe dans la continuité du traité franco-ndjuka et des différentes dispositions relatives aux Boni avec une différence essentielle : le territoire traditionnel des Saramaka est ici situé en plein Surinam. C'est sans doute l'arrêté d'investiture d'Apatou (1887) qui se rapproche le plus de l'esprit du texte. Des capitaines saramaka seront par la suite reconnus à partir de la toute fin des années 1890, des concessions éventuellement accordées.

\footnotetext{
$30 \mathrm{lbid}$. p. 56-57 et note 38, p. 122. Le document, que je n'ai pu consulter, a été trouvé par R. et S. Price aux archives départementales de Guyane et au musée des Beaux-Arts de Chartres.
}

31 II manque un verbe dans le texte. 
Dans une circulaire du 20 mars 194232, le gouvernement vichyste de la Guyane et de I'Inini écrit, au sujet des Saramaka : " l'origine de leur introduction en Guyane française, et les avantages qui leur sont accordés et remontent à 1892, résident dans une convention particulière passée avec le Grand man des Saramacas ; toutes les facilités qui leur ont été accordées ultérieurement (entrée sans pièce d'identité, sans caution de rapatriement, sans immatriculation, exemption de tous impôts et taxes), l'ont été en vue d'assurer, dans de bonnes conditions, les transports ».

C'est tout le paradoxe de cette convention : conclue par une personne physique (qui n'en signe pas moins en tant qu'ancien président du Conseil général), elle fait entrer les rapports franco-saramaka dans un phase beaucoup plus officielle. Ces rapports trouveront une expression particulièrement codifiée dans les années 1960, mais sous la forme de restitutions orales d'une convention censée avoir été adoptée en 1883.

\section{B) Par la référence à une fausse convention franco-saramaka dans les années} 1960

Dans son ouvrage pionnier, La question créole33, la sociologue Marie-José Jolivet décrit de manière détaillée le contenu d'une convention qui aurait été conclue en 1883 par le Gran Man des Saramaka avec le Gouverneur de Guyane française. Elle a expliqué récemment quelles étaient ses sources : "Ces informations mont été fournies à Régina, sur le bassin de l'Approuague, à la fin des années 1960, alors que les Marrons étaient encore sous le régime des cercles municipaux dont l'administration était le plus souvent confiée aux gendarmes des communes correspondantes. Si j'ai pu croiser mes deux sources (mémoire des Saramaka et discours de l'administrateur), je n'ai jamais eu l'accord de 1883 entre les mains. ${ }^{34}$ " La convention telle qu'elle est décrite dans l'ouvrage contient beaucoup d'invraisemblances pour un texte adopté en 1883 : référence à des fonctions et à des pratiques beaucoup plus tardives, importance accordée aux gendarmes qui porte la marque du territoire de l'Inini. Par contre, elle se réfère à des pratiques attestées par d'autres sources du coté français et du coté saramaka et constitue à ce titre un document fascinant sur le besoin de se référer à une convention écrite qui marque les relations franco-marronnes.

C'est sans doute par les précisions qu'il apporte s'agissant du rôle des capitaines saramaka pour les questions judiciaires que le document est le plus surprenant 35 : “Assisté d'un « lieutenant » qu'il choisit et qui est également nommé par le gouverneur, le " capitaine " a pour tâche de veiller à ce que la discipline soit maintenue au sein du groupe dont il a la responsabilité. Pour ce faire, il est en rapports constants avec le maire et le gendarme, mais ces derniers n'interviennent jamais directement. Dans le cas d'un litige entre deux Saramaka, c'est le " capitaine " seul qui règle la question ; lorsque le litige oppose un sujet guyanais à un Saramaka, fait d'ailleurs de mémoire d'homme extrêmement rare, le gendarme passe toujours par l'intermédiaire du " capitaine » pour résoudre avec lui le problème. Le rôle du " capitaine » sur le plan judiciaire apparaît donc comme important et délicat (...). »

\footnotetext{
32 Territoire de l'Inini, cabinet du gouverneur, circulaire, 20 mars 1942, ADGuy., dossier " Saramacca » cité dans Ibid., p. 57 et note 39.

33 Marie-José JOLIVET, La question créole. Essai de sociologie sur la Guyane française, Paris, ORSTOM, 1982, p. 154-155.

34 M.-J. JOLIVET, « Modèle occidental et créolisation. L'exemple de la Guyane », L'homme, 2013/3, $n^{\circ}$ 207-208, p. 113-134, p. 118, note 10.
}

35 « La question... », op. cit. n.32, p. 154. 
On retrouve en partie ici les principes affirmés par l'article 3 de la convention francondjuka de 1860 : compétence des Saramaka pour les conflits entre Saramaka (inter se). Par contre, alors que le texte de 1860 faisait varier la compétence en fonction du plaignant (compétence des Ndjuka pour un méfait commis par un Ndjuka à l'encontre d'un "Français ou protégé de la France ", compétence des Français pour un méfait commis par un « Français ou protégé » à l'égard d'un Ndjuka), les témoignages collectés par M.-J. Jolivet se réfèrent à un sorte de tribunal mixte composé d'un capitaine saramaka et d'un gendarme français. Pourtant, les sources dont on dispose sur ce type de conflits vont dans le sens de l'accord franco-ndjuka : ce sont les Marrons qui viennent se plaindre aux autorités françaises de méfait accomplit par un Français ou, plus largement, par un nonmarron, lesquelles interviennent en conséquence ${ }^{36}$.

C'est sans doute parce qu'ils se sont très tôt organisés en diaspora tout en faisant en sorte de conserver leur spécificités, parce qu'ils ont su jouer des rivalités entre puissances, que les peuples marrons du Surinam persistent aujourd'hui et gagnent même en dynamisme. Si ces peuples sont aujourd'hui en mutation en raison de la naissance d'un nouveau type de relation entre la côte et leur territoire traditionnel, les conventions franco-marronnes montrent que les autorités françaises peuvent aussi être obligées, en retour, de se marronniser...

36 Pour l'Inini, cf. « Un projet politique... », op. cit. n.15, p. 551ss.

On n'a pas souvenir de plainte concernant un méfait commis par un Marron à l'égard d'un Français, si l'on met de coté les habituelles récriminations contre les capitaines et chefs coutumiers.

Les textes relatifs à l'organisation de la justice dans l'Inini ne contiennent aucune disposition relatives aux Amérindiens et aux Marrons. 